\title{
The Concept of Building a Network of Digital Twins to Increase the Efficiency of Complex Telecommunication Systems
}

\author{
Sh. Zh. Seilov ${ }^{D},{ }^{1}$ A. T. Kuzbayev, ${ }^{1}$ A. A. Seilov, ${ }^{1}$ D. S. Shyngisov, ${ }^{1}$ V. Yu. Goikhman, ${ }^{2}$ \\ A. K. Levakov, ${ }^{3}$ N. A. Sokolov, ${ }^{4}$ and Y. Sh. Zhursinbek ${ }^{1}$ \\ ${ }^{1}$ L. N. Gumilyov Eurasian National University, Nur-Sultan, Kazakhstan \\ ${ }^{2}$ Bonch-Bruevich Saint-Petersburg State University of Telecommunication, LLC "Scientific and Technical Center SOTSBI", \\ Saint-Petersburg, Russia \\ ${ }^{3}$ JSC "Iskrauraltel", Ekaterinburg, Russia \\ ${ }^{4}$ LLC Scientific and Technical Center "Protei ST", Saint-Petersburg, Russia
}

Correspondence should be addressed to Sh. Zh. Seilov; seilov_shzh@enu.kz

Received 15 June 2020; Revised 28 August 2020; Accepted 1 March 2021; Published 15 March 2021

Academic Editor: Rosa M. Lopez Gutierrez

Copyright (c) $2021 \mathrm{Sh}$. Zh. Seilov et al. This is an open access article distributed under the Creative Commons Attribution License, which permits unrestricted use, distribution, and reproduction in any medium, provided the original work is properly cited.

\begin{abstract}
The technical literature actively discusses ideas for creating digital twins to solve a wide range of problems that arise for enterprises of various kinds. Advances in the areas of information technology and the development of the necessary software really make it possible to obtain important new results through the creation of digital twins. This paper proposes the concept of building a network of digital twins, used to solve a number of actual problems in complex telecommunication systems. Two applications are considered examples of such tasks. The first application is the organization of information feedback for enterprises involved in the life cycle of a telecommunication network: "elaboration of modernization principles, development of equipment, design, construction, and operation." The second application is monitoring traffic, including its atypical behavior, in order to avoid the recurrence of erroneous actions in case of emergency situations. Another important feature of the digital twin network is that it will become an effective tool for conducting interdisciplinary research.
\end{abstract}

\section{Introduction}

Using the functionality of the cybernetic model [1], called the digital twin, allows us to solve a number of complex problems of various kinds. The difference between the digital twin and the conventional mathematical models [2] is that it allows us to very accurately display the current state of the investigated object or process. At the same time, the digital twin can not only operate with the reality that is supposed to exist objectively but also work with augmented reality [3] and with an altered reality. The concept "altered reality" was proposed in [4].

This feature of the digital twin is illustrated in Figure 1. The dotted line under the number 3 indicates the relationship between augmented and altered reality. The functionality of this connection is the subject of a separate study. Features of the digital twin, as applied to one object, are well described in the technical literature [5-7].

In 2003, Michael Grieves first proposed the digital twin terminology as "digital equivalent to a physical product" [8]. However, up till now, no formal or widely accepted definition has been achieved, and no unified creating and deploying process, too. Lots of digital twin conceptual models or reference models have been proposed. And different understandings about digital twin and digital twin conceptual models are reviewed by Liu et al. [9]. For example, Grieves and Vickers [10] defined two types of digital twins: digital twin prototype (DTP) and digital twin instance (DTI), and DTs were in a digital twin environment (DTE). Bao et al. [11] defined three types of digital twin models from the perspective of the manufacturing process on the shop floor, which were Product Digital Twins, Process Digital 


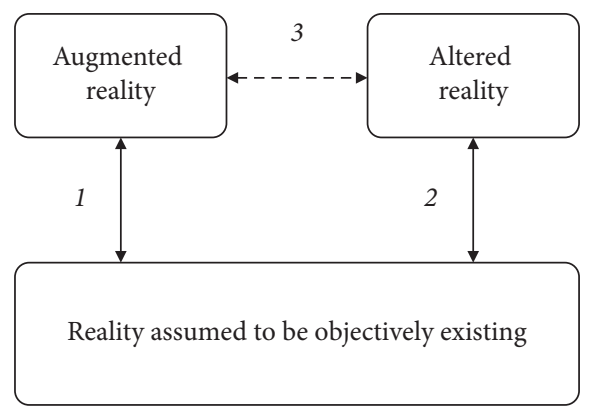

Figure 1: Two types of realities.

Twins, and Operation Digital Twins. Ullah [12] proposed three types of digital twins: object twins, process twins, phenomena twins.

The digital twin model must contradic between the simplified virtual model and the complex behavior of the physical object [9]. A variety of frameworks and reference models of digital twin were proposed, but none of them become industry consensus. Some researches adopt an information management-oriented view, focusing on semantic connections and seamless information flow [12]. A compromised approach is to implement flexible modeling in a modular way. Negri et al. [13] proposed adding black-box modules to the main simulation model. Black-box modeling using data or grey-box modeling using a combination of physics and data is also feasible.

Digital twin is a base concept of Industry 4.0 manufacturing. In complex cyber-physical production systems with many digital twins, information must be aggregated. This leads to the need for digital twins to interact. Therefore, the network models for twin-to-twin communication are needed.

The purpose of this paper is to develop a concept that extends the principles of applying digital twins to a set of objects or processes. The main idea is to create a network that unites various digital twins. A complex telecommunication system that provides support for a wide range of services was chosen as an example of such an aggregate.

The proposed concept is aimed at ensuring the sustainable functioning of complex telecommunication systems. The developed solutions are illustrated by two main examples.

The first example is related to the organization of a feedback chain for the main participants of the telecommunications market engaged in the life cycle, which is represented by the following set of operations:

(i) Formation of principles for the operating telecommunication network modernization.

(ii) Development of equipment for the transmission, switching, and processing of information.

(iii) Preparation of design documentation for the work, allowing us to begin the modernization of the operating telecommunication network.

(iv) Carrying out construction work, as a result of which the telecommunication system as a whole is updated. (v) Organization of technical operation processes taking into account new properties of the telecommunication system.

The second example is related to monitoring of served traffic. For this example, we consider a special case concerning a radical change in traffic due to emergency situations. Monitoring traffic in such situations has shown that a sharp increase in the activity of users of the telecommunication system begins. If it is possible to find rational solutions for traffic service in such cases, then the relevant experience must be used by all telecommunication operators.

The idea of creating a digital twin network, as far as the authors know, is new. For this reason, real projects aimed at building a network of digital twins are not presented in the paper.

The rest of this paper is structured as follows. Section 2 analyzes the features of mathematical models of a digital twin. The next section contains proposed conceptual provisions for creating a network of digital twins. The two examples of the application of this concept mentioned above are described in the fourth and fifth sections, respectively. Section 6 presents considerations regarding the possibility of using a network of digital twins to predict the behavior of complex systems in emergency situations of various natures. Analysis of the nature of information processes in a network of digital twins is the subject of the seventh section. Conclusion and future research are presented in Section 8.

\section{Features of Mathematical Models}

One of the commonly used mathematical models is a black-, grey-, or white-box [1,2]. A black-box is considered an object, the essence of which we do not know anything except for a set of functions of the form $A(t), B(t), C(t)$, and $D(t)$. These functions describe the external action and operations performed by the black-box, control actions, and the result, respectively. For the grey-box, an example of which is shown in Figure 2; in addition to knowing the functions $A(t), B(t)$, $C(t)$, and $D(t)$, incomplete information on the structure and functions of the investigated object is available. A white-box model is used in cases where information about the structure and functions of the studied object is considered complete and reliable.

An important feature of this model is that it implements a feedback loop. It often allows us to significantly increase the value of the model. Unfortunately, in practice, it is not always possible to create a model in which there is a feedback loop. One of the tasks of the digital twin is to build an effective feedback loop that can change its functions, taking into account the features of the processes occurring in the telecommunication system.

The effectiveness of using mathematical models is determined by a number of factors, among which it is appropriate to single out three important points:

(a) The degree of adequacy of the proposed model to the studied object or process

(b) The accuracy of the mathematical operations performed during the study of the model 


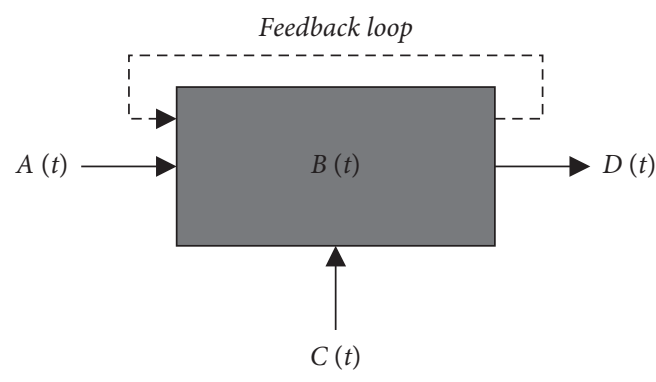

Figure 2: Model of "grey-box."

(c) The correct interpretation of mathematical results for practical decisions

Figure 3 shows three phases: the development of the model, its study, and interpretation of the results. At each phase, processes that lead to the accumulation of errors are realized. As a result, a certain dependence $F_{1}\left(x_{1}, x_{2}, x_{m}\right)$, which can conditionally be considered correct, is gradually transformed into the function $F_{2}\left(y_{1}, y_{2}, y_{n}\right)$. This function contains a number of errors that can significantly distort the necessary results.

It should be noted that the achievements of modern mathematics allow us to state that the minimal errors in the study of the model correspond to point (b). Minimization of errors determined by point (a) is ensured by using the model in the form of a digital twin. The degree of adequacy may approach the maximum possible level. To a large extent, the same conclusion applies to point (c). However, the validity of this statement is associated with the use of a model in the form of a network of digital twins. This hypothesis is considered in the next section.

\section{Digital Twin Network Model}

At first glance, the proposed model is formed as a simple collection of digital twins. In fact, the digital twin network is a more complex design. Figure 4 shows a model consisting of seven digital twins. Each digital twin serves as a model of one object. A typical example of such an object is a switching node. Interfaces between model elements are designated as $\mathrm{NNI}_{J}$, Network Node Interface of the $j$ th type. The given illustration contains three types of $\mathrm{NNI}_{J}$ interfaces, $\mathrm{A}, \mathrm{B}$, and C.

The difference in interfaces is determined mainly by the stack of protocols used [13]. At the Network Access Layer, Ethernet technology is typically used. At the Internet and Transport layers, the TCP/IP protocol stack is usually dominant. At the Application layer, most likely, the development of a new protocol or even a set of protocols is required. It can be argued that Big Data [14], Data Mining [15], Neural Networks [16], and the like will be widely used in a network of digital twins. Obviously, in a network of digital twins, both cloud and fog computing will be widely used $[17,18]$.

Information accumulated by a network of digital twins will become a good base for scenarios for the development of telecommunication networks [19]. Processing this

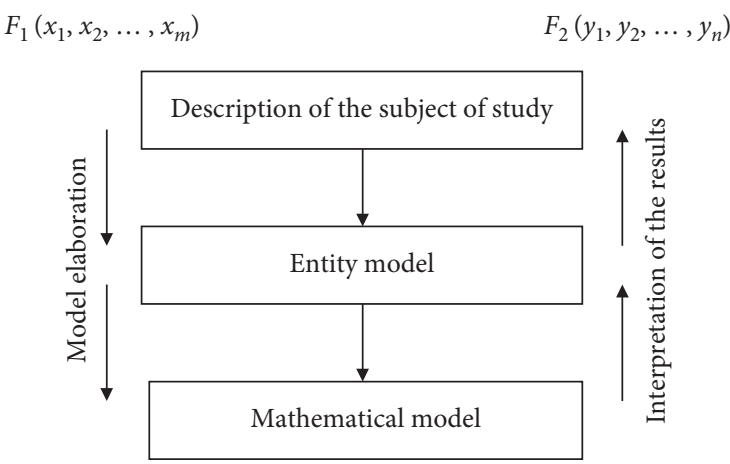

Figure 3: Stages of possible error accumulation.

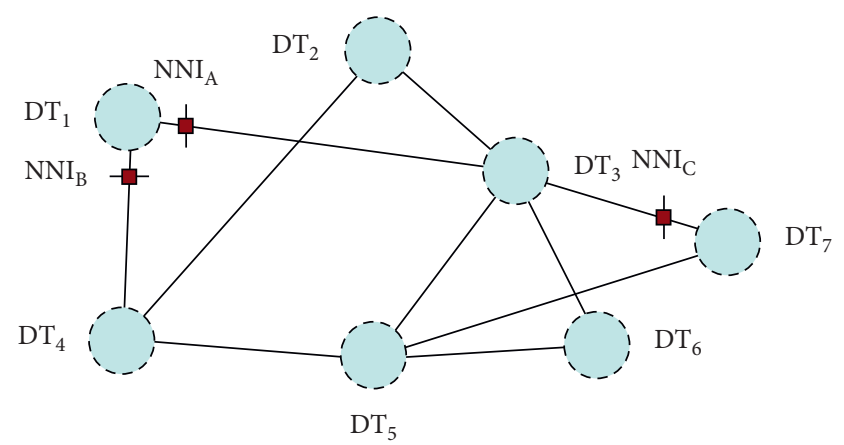

FIgURE 4: Network of the digital twins.

information will reduce the risks [20], inevitable for the development of complex systems of any kind due to the uncertainty of the driving forces of evolutionary processes.

An assertion of this kind should be illustrated by several examples. Two such examples are discussed in the following sections of the paper.

\section{Digital Twin Network and Feedback Loops}

The lifecycle of a telecommunication system can be represented in the form of five successive stages, shown in Figure 5. It is assumed that the end of the life cycle of a telecommunication system is a qualitatively new stage in its modernization. This circumstance and the limitation of the number of stages explain the use of the word "simplified" in the name of the proposed model.

Set $W_{1}$ includes a set of theoretical research results that form the concept of creating or upgrading a telecommunication system.

The values of the remaining sets $W_{k}(k=2,3,4,5)$ are determined by the names of the corresponding rectangles.

Some of the results are used by developers of the transmission, switching, and processing equipment. At the same time, some theoretical provisions are being revised. The measure of the difference in solutions for steps $i$ and $j$ $(j=i+1)$ is denoted as $P_{i j}$. The $Q_{i j}$ value is considered a measure of the effectiveness of feedback between solution developers for steps $i$ and $j(j=i-1)$.

An analysis of the main stages of the lifecycle carried out for a number of telecommunication systems showed that the 


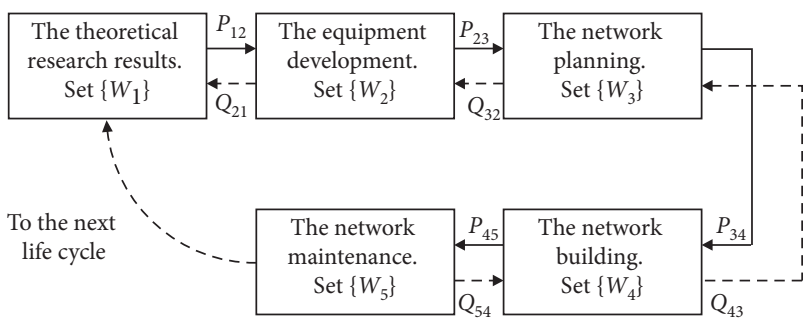

Figure 5: Simplified lifecycle of the telecommunication network.

differences between $P_{i j}$ are very significant, and the feedback efficiency between solution developers is close to zero. A radical change in the current situation can be ensured by creating a network of digital twins. This network will allow the solution developer to track all changes made in the subsequent stages and intervene in the development of the telecommunication system. In other words, the values of $P_{i j}$ can be minimized. Automation of information exchange provides maximization of $Q_{i j}$ values. There are no quantitative estimates of such an analysis yet. Apparently, a network of digital twins will help get the needed results.

\section{Digital Twin Network and Traffic Monitoring}

The results of traffic monitoring serve as an important information base for the modernization of the telecommunication system [21]. For the implementation of traffic monitoring functions, both software of switching nodes and specialized tools can be used [22]. The quality of service indicators for a telecommunication system is determined by the recommendations of the International Telecommunication Union [23]. The task of all the main participants in the telecommunications market is to provide normalized indicators of the quality of service for multiservice traffic [24].

Figure 6 shows a model of a telecommunication system (left fragment) and its digital twin (right fragment). It is assumed that the communication network consists of five routers. Consequently, the network of digital twins will have five elements. A network of digital twins is an undirected graph with vertices $a_{i}$ and edges $b_{i j}$. The terms used in the proposed construction are borrowed from the concept of dia\$par [25]. This system includes the concept of "digital twin" as one of the main components.

The vertices $a_{i}$ and the edges $b_{i j}$ can be associated with various metrics or their combination. Typical examples of metrics are the cost of the hardware, throughput indicators, and reliability characteristics. From the point of view of the considered example, the main interest is associated with the characteristics of the served traffic, but the cost and reliability metrics can also be claimed.

The results accumulated by the network of digital twins are useful for solving many problems. A very important aspect of traffic monitoring is its behavior in emergency situations [26]. Such cases lead to avalanche-like growth of traffic. This process can be aggravated by the failure of parts of the equipment that occur due to the destructive effects inherent in emergency situations. In addition, the list of

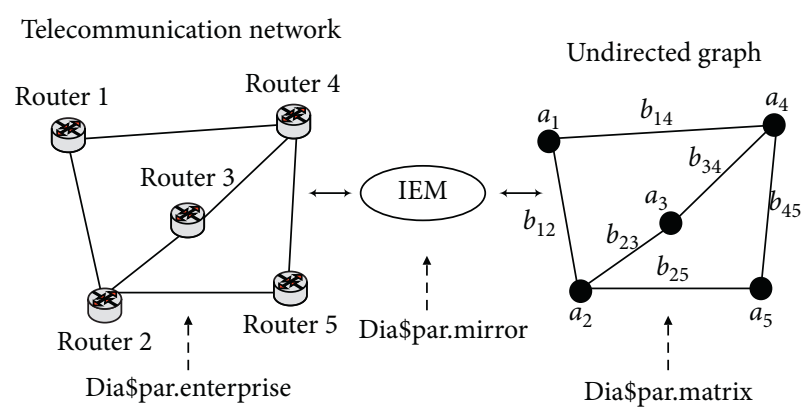

IEM, Intelligent Enterprise Managing

FIgURE 6: Digital twin creation for telecommunication network.

services demanded by users of the telecommunication system may change significantly.

The prototype of the network of digital twins made it possible to solve a number of urgent problems regarding the functioning of the multiservice network in emergency situations $[27,28]$. The results obtained indicate the advisability of using feedback loops, an example of which was given in the previous section.

\section{Digital Twin Network and Prediction of the Behavior of Complex Systems in Emergency Situations}

Emergency traffic monitoring, mentioned in the previous section, is an example of passive use of the functionality that is typical for a digital twin network. Additional properties of a network of digital twins that are not explicitly inherent in complex systems can be used to predict their behavior in the event of atypical situations of various kinds. Besides, a network of digital twins can potentially work with unstructured data. This issue is briefly discussed in the next section.

One of the typical examples of an atypical situation is the outlier of the observed values of a process. In statistics, the observed value is considered an outlier, which differs markedly from the rest of the sample elements [29]. Outliers, as a rule, arise randomly. They can be caused by measurement errors and also testify to the presence of the socalled "heavy-tailed distribution" [30]. Also, other reasons for the appearance of outliers are possible.

Figure 7 shows the behavior of a certain function $f(x)$, for which it is appropriate to distinguish between three ranges along the abscissa. The first range includes the values of the function in question on the interval $\left[0, x_{1}\right]$. On the interval $\left(x_{1}, x_{2}\right)$, no values of the function $f(x)$ are observed. Recent changes in the function $f(x)$ are observed on the interval $\left[x_{2}, x_{3}\right]$.

Similar situations can be found when measuring Internet traffic. They are similar to the Tukey-Huber model [31], for which, with a very low probability $\varepsilon$, the investigated process $f(x)$ is represented by the function $h(x)$. Moreover, with a high probability equal to $(1-\varepsilon)$, a process is observed that is described by the function $g(x)$ : 


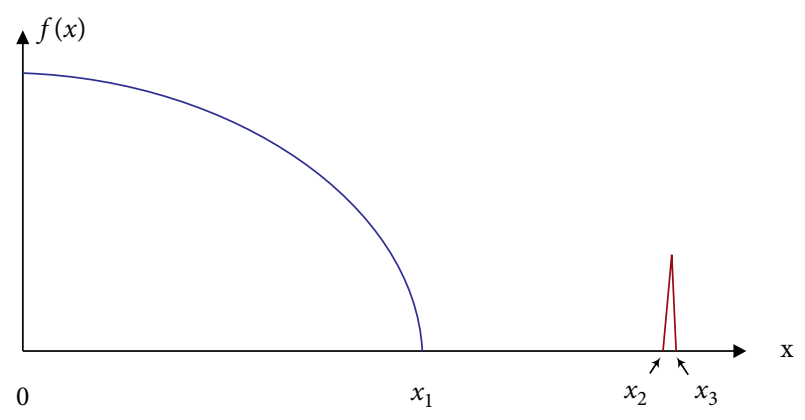

FIgURE 7: Example of the behavior of the function $f(x)$.

$$
f(x)=(1-\varepsilon) g(x)+\varepsilon h(x) .
$$

In a network of digital twins, it is possible to conduct experiments that are difficult to carry out, using real objects, without serious problems for ongoing business processes. For example, the task was set to verify the quality of the telecommunications network in conditions of significant traffic growth. If such traffic is artificially created, then there will be a problem in the telecommunication network with the quality of customer service, which can lead to complaints and the need to pay fines to those users who have entered into a Service Level Agreement with a service provider [32]. In a network of digital twins, an experiment with a sharp increase in traffic will not lead to negative consequences.

The use of the Tukey-Huber model and similar ones will allow us to develop prognostic estimates necessary to choose the path of modernization of complex systems. It should be emphasized that forecasts based on the use of a network of digital twins usually provide an answer to the question: "What will happen?" A reasonable question "Why can this happen?" requires independent research, for which a network of digital twins will not always be an effective tool. An acceptable level of efficiency can be achieved if the initially complex system is built on the basis of a rational solution [33]. In this case, a complex system stays stable when changing external and internal factors affecting the stability of its functioning.

It is possible that, in order to solve forecasting problems, some models in the network of digital twins will have to be perfected. The complexity of the refinement will depend on the nature of the complex system for which a network of digital twins is being created. Moreover, in some cases, the collaboration of two or more networks of digital twins will be useful.

To illustrate the last statement, we can give an example, which is sometimes given by specialists in the Big Data and Data Mining technologies $[14,15]$. At one enterprise, from time to time, mass failures occurred in electronic units, which were assembled manually. For a long time, it was not possible to identify the pattern of occurrence of failures. Then one of the specialists drew attention to the fact that the installation of these blocks was carried out mainly by young women. An analysis of the periods of time during which the failures were observed and the late pregnancy of employees engaged in the installation of electronic components made it possible to establish the pattern. Increased sweating during assembly affected the quality of the contacts, which led to block failures. After pregnant employees were transferred in advance to other operations before going on maternity leave, the refusals ceased.

To obtain such results, it should be possible to combine those networks of digital twins that are initially created to solve completely different problems. Thus, we can say that we should focus on combining networks of digital twins, which is a very difficult task. As a result of such a combination, a kind of super network of digital twins is created.

\section{Information Processes in the Digital Twin Networks}

A network of digital twins is a specific information system [34]. Some types of information may be represented by structured data. Processing such data is a relatively simple task. Part of the information should be considered unstructured data. Their processing is usually more complicated. Apparently, for a network of digital twins, it will be necessary to develop standards that define the rules for creating and processing information.

For those objects and processes that often change due to the introduction of innovative solutions, standards for the creation and processing of information will have to be reviewed periodically. For this reason, it is necessary to develop a methodological approach that will allow us to quickly and cost-effectively update the relevant software.

One of the possible approaches was proposed in [35]. It was developed to solve problems that are significantly influenced by singularity processes [36]. These processes should be considered when building and developing digital twin networks. For this reason, the methodological approach described in [35] is also interesting for minimizing the costs of supporting information processes in digital twin networks.

In a simplified form, the essence of the methodological approach is shown in Figure 8. Equipment for building a network of digital twins can be represented in the form of two components due to the decomposition operation: hardware and software. Further, each of these components is divided into invariable core and variable component. When developing equipment for a network of digital twins, one should strive to minimize the costs of variable components (for both hardware and software). This fact is indicated by an arrow pointing down. It is possible that, at the initial stage of creating a network of digital twins, this will require an increase in costs for the invariable core, which is indicated by the arrow pointing up.

A certain increase in costs at the initial stage of creating a network of digital twins will be compensated by the high speed of introducing new functionalities, aimed primarily at the adequacy of the model to its original. For the practical implementation of the proposed solution, a very important condition is the prediction of further development paths of the studied objects or processes. For this purpose, various forecasting methods can be used [37]. Along with prognostic 


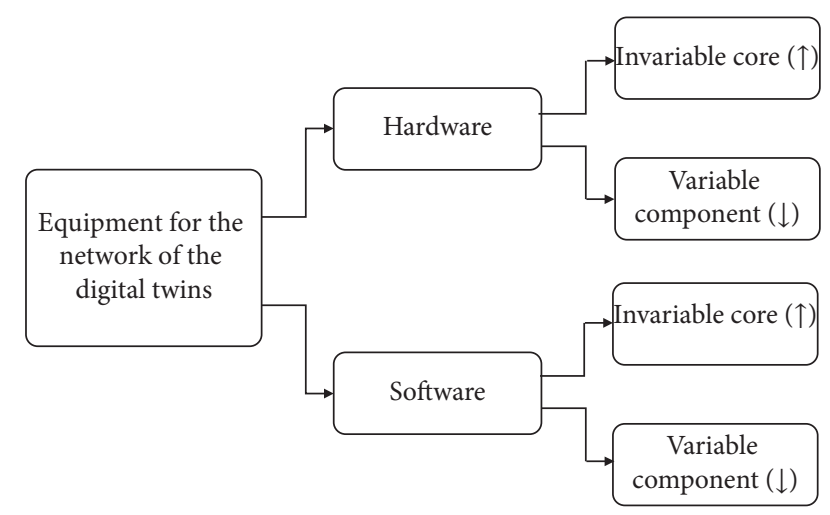

FIgURE 8: Decomposition of equipment for the network of digital twins.

estimates, various scenarios for the further development of the studied objects or processes should be developed [19].

The data exchanged by digital twins include messages of varying degrees of importance. In addition, they can vary markedly in volume. Sometimes, to identify an event, it is enough to transmit one bit, and in some cases, we have to exchange video with a volume that is estimated in megabytes and even gigabytes. Obviously, for the majority of digital twins, the resources of public telecommunication networks will be used. For this reason, additional traffic can noticeably worsen the quality of service indicators established by applicable standards.

The emerging problem can be solved in various ways. One of them is the rational assignment of priorities [38] to different types of traffic in a network of digital twins. As an example, consider Figure 9 [39], which shows the dependences of the average waiting time $W_{i}^{(1)}$ for demands of different priority in the load range of the teletraffic system $\rho$ from 0 to 0.6 . The demand is considered an IP packet. The subscript of the value $W_{i}^{(1)}$ determines the priority level. If $i=0$, then all applications are served without priorities in the order they are received at the entrance of the teletraffic system.

The curves are constructed for the relationship between the intensity values of demands of the first, second, and third priorities, defined as follows: 10,30 , and $60 \%$.

The dashed line shows the dependence of the average duration of waiting for applications when serving them without priorities. All values $W_{i}^{(1)}$ are normalized to the average time of their service (processing of IP packets in a router or transmission over information exchange paths).

The course of the curves clearly demonstrates that the introduction of a priority service discipline can significantly improve the quality of service indicators for some types of services. For example, with the introduction of three priorities, the average waiting time for applications of the first priority is noticeably reduced. It should be emphasized that, for multiservice networks based on packet technologies, the priority discipline of service is recommended by the International Telecommunication Union [40].

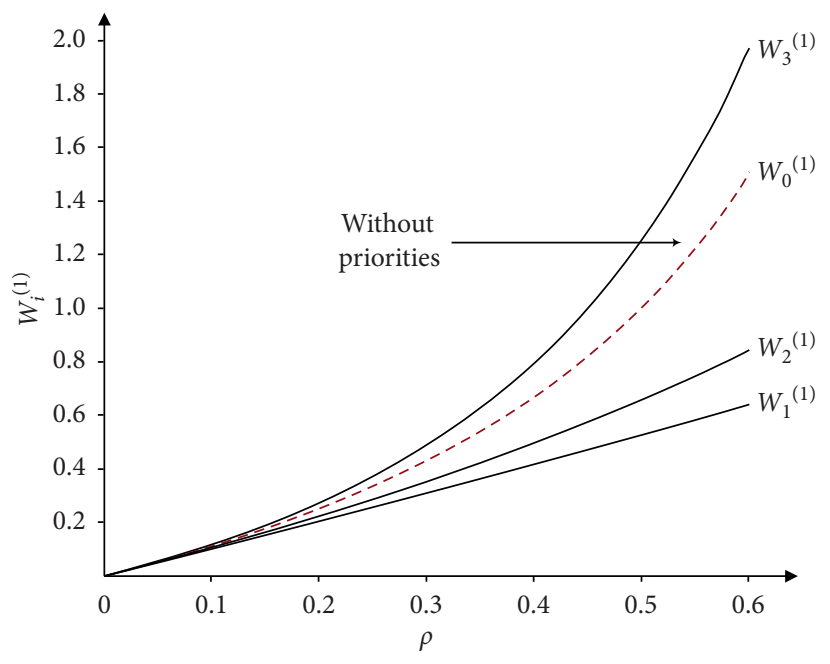

FIgURE 9: Mean values of waiting time for demands with different priorities.

There are several factors to consider when prioritizing IP packets that transmit information in a digital twin network. They can be divided into two large groups, internal and external. A typical example of internal factors is the importance of information in terms of decision-making time to resolve a problem. The effect of the additional load on the public telecommunications network is an important external factor that should be considered when choosing the priority level for IP packets related to the work of digital twins.

The actual utilization of the resources of the public telecommunications network should also be taken into account. In some cases, these resources are redundant. This situation is usually typical after the completion of the next stage of the modernization of the telecommunication network. Then, some IP packets that transmit information in a digital twin network can be assigned the first or second priority level. This statement is true if the specified quality indicators are provided for those subscribers who have concluded the so-called Service Level Agreements with the Telecommunications Operator [41].

The queuing theory is based on a number of assumptions that are not always correct for real telecommunications networks. A network of digital twins can accomplish two interrelated challenges. First, it does not introduce assumptions that can significantly affect the accuracy of the problem solution. Secondly, it helps to find new approaches to solving complex problems in the field of teletraffic theory.

Over time, the resources of a network built with some redundancy come in line with the served traffic. Then the first and second priorities for IP packets that carry information in a network of digital twins can be assigned very rarely. Such a solution is possible for a minimum number of such IP packets. Apparently, priority service is generally acceptable for short messages of high importance. The main traffic generated in the digital twin network will be served with a lower priority level. 
An alternative option for building a network of digital twins is renting the necessary resources from the Telecommunications Operator or building dedicated information and communication system. This approach will be acceptable for objects of special importance since it is associated with very high investments.

\section{Future Research Directions}

It should be emphasized that the creation of digital twins does not negate the relevance of serious scientific research. Digital twins only make it possible to increase the efficiency of scientific research through the use of models that are as adequate as possible to the objects and processes under consideration.

Historically, digital twins are associated by some experts with an approach called the BIM, Building Information Model [42]. The similarity between BIM and the digital twin lies mainly in the general principles of mapping real objects and processes to the world of models. The true value of digital twins, as well as the dia\$par concept, will be manifested in conducting interdisciplinary studies [43]. They are considered by the authors of this paper as one of the most important areas of further research. The digital twin network mentioned above is designed to solve problems that require the use of multidisciplinary approaches. Among interdisciplinary works, it is appropriate to note the following publications [44-46].

The second line of further research is aimed at implementing the concept of "Society 5.0" [47], also called Super Smart Society. One of the important provisions of "Society 5.0 " is the widespread use of promising digital technologies. This fact allows us to consider the practical application of digital twins as an integral system solution for the concept of "Society 5.0."

As the third area of further research, the development of economic and mathematical methods for the study of models presented in the form of digital twins should be mentioned. Such methods seem to be very important and cannot be replaced by other ones of obtaining new knowledge. On the other hand, new methods will be used in conjunction with perspective solutions that expand.

Standard methods of teletraffic models investigate with queues $[48,49]$. In conclusion, it is appropriate to formulate the influence of a network of digital twins on the chain "Data-Information-Knowledge-Wisdom" [50]. The corresponding concept is known by the abbreviation DIKW. Figure 10 shows an additional layer, "Understanding," which is used by some DIKW researchers.

Most likely, the network of digital twins will most of all influence three layers in the chain "Information-Knowledge-Understanding." The degree of positive influence will vary. According to the authors of this paper, the most significant influence will fall on the "Understanding" layer.

This assumption is true at the current level of artificial intelligence [51]. It is possible that in the future a network of digital twins will also create such results that could be attributed to the Wisdom layer.

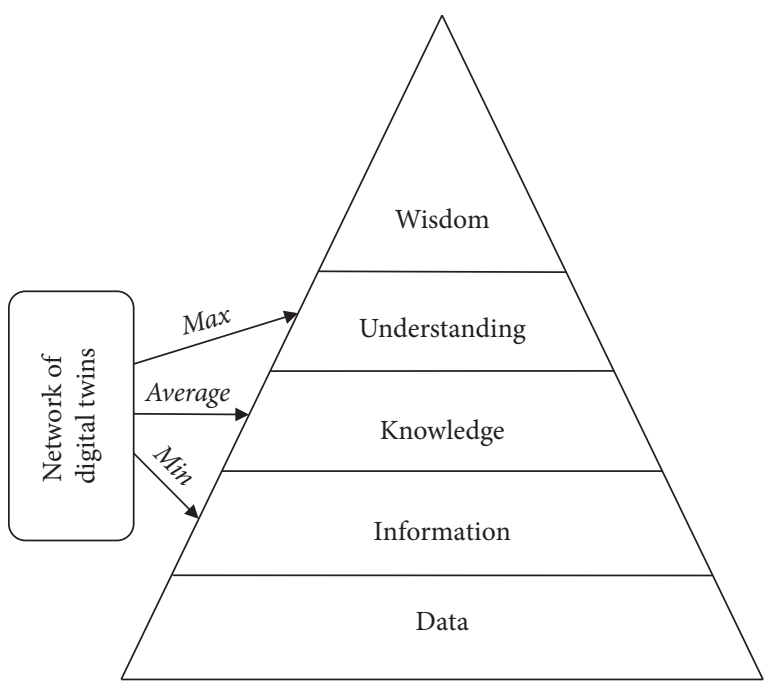

FIgURE 10: Network of digital twins and DIKW concept.

\section{Data Availability}

The data used to support the findings of this study are included in the article.

\section{Conflicts of Interest}

The authors declare that they have no conflicts of interest.

\section{Acknowledgments}

This work was supported by the Ministry of Education and Science of the Kazakhstan Republic under grant AP05134349.

\section{References}

[1] W. R. Ashby, An Introduction to Cybernetics, Martino Fine Books, Torrington, CT, USA, 2015.

[2] A. D. Mishkis, Elements of the Theory of Mathematical Models, KomKniga, Moscow, Russia, 2007.

[3] K. J. Varnum, Beyond Reality: Augmented, Virtual, and Mixed Reality in the Library, ALA Editions, Chicago, IL, USA, 2019.

[4] A. K. Levakov and N. A. Sokolov, Concept "Altered Reality", Vestnik Sviazy, 2018.

[5] E. A. Michael and B. Kalyan Ram, "Cyber-physical systems and digital twins," in Proceedings of the 16th International Conference on Remote Engineering and Virtual Instrumentation, Springer, Cham, Switzerland, February 2020.

[6] M. Farsi, A. Daneshkhah, A. Hosseinian-Far, and H. Jahankhan, Digital Twin Technologies and Smart Cities (Internet of Things), Springer, Berlin, Germany, 2019.

[7] Digital Twin, Driving Business Value throughout the Building Life Cycle, Siemens White Paper. Siemens Switzerland Ltd., Switzerland, 2018.

[8] M. Grieves, Digital Twin: Manufacturing Excellence through Virtual Factory Replication, White Paper, Switzerland, 2014.

[9] M. Liu, S. Fang, H. Dong, and C. Xu, "Review of digital twin about concepts, technologies, and industrial applications," Journal of Manufacturing Systems, vol. 58, pp. 346-361, 2020. 
[10] M. Grieves and J. Vickers, "Digital twin: mitigating unpredictable, undesirable emergent behavior in complex systems," in Transdisciplinary Perspectives on Complex Systems, F. J. Kahlen, S. Flumerfelt, and A. Alves, Eds., pp. 85-113, Springer, Cham, Switzerland, 2017.

[11] J. Bao, D. Guo, J. Li, and J. Zhang, "The modelling and operations for the digital twin in the context of manufacturing," Enterprise Information Systems, vol. 13, no. 4, pp. 534-556, 2019.

[12] A. Sharif Ullah, "Modeling and simulation of complex manufacturing phenomena using sensor signals from the perspective of Industry 4.0," Advanced Engineering Informatics, vol. 39, pp. 1-13, 2019.

[13] E. Negri, L. Fumagalli, C. Cimino, and M. Macchi, "FMUsupported simulation for CPS digital twin," Procedia Manufacturing, vol. 28, pp. 201-206, 2019.

[14] T. Erl, W. Khattak, and P. Buhler, Big Data Fundamentals: Concepts, Drivers \& Techniques, Prentice-Hall, Upper Saddle River, NJ, USA, 2015.

[15] J. Han, M. Kamber, and J. Pei, Data Mining Concept and Techniques, Morgan Kaufmann Publishers, Burlington, MA, USA, 2011.

[16] C. C. Aggarwal, Neural Networks and Deep Learning, Springer, Berlin, Germany, 2018.

[17] T. Erl, Z. Mahmood, and R. Puttini, Cloud Computing: Concepts, Technology \& Architecture, Prentice-Hall, Upper Saddle River, NJ, USA, 2013.

[18] M. Chiang, B. Balasubramanian, and F. Bonomi, Fog for 5G and IoT, Wiley, New York, NY, USA, 2017.

[19] W. R. Huss, "A move toward scenario analysis," International Journal of Forecasting, vol. 4, no. 3, pp. 377-388, 1988.

[20] C. Yoe, Principles of Risk Analysis: Decision Making under Uncertainty, CRC Press, Boca Raton, FL, USA, 2011.

[21] ITU-D, "Teletraffic engineering handbook," in International Telecommunication Union, V. B. Iversen, Ed., Telecommunication Development Bureau, Geneva, Switzerland, 2003.

[22] V. Goichman, K. Esalov, and N. Sokolov, "Using specialized computer systems to study the characteristics of telecommunication networks," in Proceedings of the FRUCT'18, pp. 456462, ITMO University, Saint-Petersburg, Russia, April 2016.

[23] ITU-T, Definitions of Terms Related to Quality of Service, ITU, Geneva, Switzerland, 2008.

[24] M. Barreiros and P. Lundqvist, QOS-enabled Networks: Tools and Foundations, Wiley, Hoboken, NJ, USA, 2016.

[25] https://diasparbusiness.com/cis-ru/diaspar-basics/.

[26] ITU-T, Next Generation Networks-Emergency Telecommunications-Technical Considerations, ITU, Geneva, Switzerland, 2011.

[27] A. Levakov and N. Sokolov, "Access to Emergency services during overload traffic period," in Proceedings of the 12th International Conference "Internet of Things, Smart Spaces, and Next Generation Networking", Springer, St. Petersburg, Russia, August 2012.

[28] A. K. Levakov and N. A. Sokolov, "Three algorithms for traffic limitation in emergencies," in Proceedings of the Selected Papers of the IX Conference "Information and Telecommunication Technologies and Mathematical Modeling of HighTech Systems", Moscow, Russia, February 2019.

[29] F. E. Grubbs, "Procedures for detecting outlying observations in samples," Technometrics, vol. 11, no. 1, pp. 1-21, 1969.
[30] S. Foss, D. Korshunov, and S. Zachary, An Introduction to Heavy-Tailed and Subexponential Distributions, Springer, Berlin, Germany, 2013.

[31] J. W. Tukey, A Survey of Sampling from Contaminated Distributions. Contributions to Probability and Statistics, Stanford University Press, Palo Alto, CA, USA, 1960.

[32] W. Fawaz, B. Daheb, O. Audouin, M. Du-Pond, and G. Pujolle, "Service level agreement and provisioning in optical networks," IEEE Communications Magazine, vol. 42, no. 1, pp. 36-43, 2004.

[33] A. Sokolov and N. Sokolov, "Rational solutions for development of telecommunications networks," T-Commerce, vol. 8, no. 6, pp. 81-84, 2014.

[34] R. Stair and G. Reynolds, Fundamentals of Information Systems, Cengage Learning, Boston, MA, USA, 9th edition, 2017.

[35] N. A. Kulikov, A. V. Pinchuk, and N. A. Sokolov, "Development features of innovative solutions for the long term," First Mile, vol. 5, pp. 48-53, 2019.

[36] A. H. Eden, J. H. Moor, J. H. Soraker, and E. Steinhart, Singularity Hypotheses. A Scientific and Philosophical Assessment, Springer-Verlag, Berlin, Germany, 2013.

[37] R. Hyndman and G. Athanasopoulos, Forecasting: Principles and Practice, OTexts, Melbourne, Australia, 2013.

[38] D. Gross, J. F. Shortle, M. James, J. M. Thompson, and C. M. Harris, Fundamentals of Queueing Theory, Wiley, Hoboken, NJ, USA, 5th edition, 2018.

[39] A. N. Sokolov, N. A. Sokolov, and V. S. Zaitsev, Teletraffic Systems with One Server, University of Telecommunications, St. Petersburg, Russia, 2019.

[40] ITU-T, Network Performance Objectives for IP-Based Services, ITU, Geneva, Switzerland, 2011.

[41] J. Desai, Service Level Agreements: A Legal and Practical Guide, ITGP, Oxford, UK, 2010.

[42] R. Sacks, C. Eastman, C. Lee, and P. Teicholz, BIM Handbook: A Guide to Building Information Modeling for Owners, Designers, Engineers, Contractors, and Facility Managers, Wiley, Hoboken, NJ, USA, 2018.

[43] R. Frodeman, J. T. Klein, and R. C. Dos Santos, The Oxford Handbook of Interdisciplinarity, Oxford University Press, Oxford, UK, 2019.

[44] P. Stavropoulos, A. Papacharalampopoulos, and L. Athanasopoulou, "A molecular dynamics based digital twin for ultrafast laser material removal processes," International Journal of Advanced Manufacturing Technology, vol. 108, pp. 413-426, 2020.

[45] https://developer.ibm.com/technologies/iot/articles/whatare-digital-twins/.

[46] Y. Lu, "Digital Twin-driven smart manufacturing: connotation, reference model, applications and research issues," Robotics and Computer-Integrated Manufacturing, vol. 61, Article ID 101837, 2020.

[47] B. Salgues, Society 5.0: Industry of the Future, Technologies, Methods and Tools, Wiley, Hoboken, NJ, USA, 2018.

[48] V. Goikhman, S. Seilov, N. Sokolov, and L. Korganbayeva, "Evaluating the packet traffic parameter measurements," Telecommunications and Radio Engineering, vol. 78, no. 6, pp. 489499, 2019.

[49] S. Seilov, V. Goikhman, V. Goykhman, M. Kassenova, A. Seilov, and D. Shingissov, "Development of neural network models for the analysis of infocommunication traffic," Bulletin of the Karaganda University-Mathematics, vol. 96, no. 4, pp. 118-126, 2019. 
[50] R. L. Ackoff, "From data to wisdom," Journal of Applied Systems Analysis, vol. 16, pp. 3-9, 1989.

[51] S. Russell and P. Norvig, Artificial Intelligence: A Modern Approach, Pearson, London, UK, 2016. 